ARKIV FÖR MATEMATIK Band 3 nr 23

Mitgeteilt am 14. September 1955 durch T. NAGELL und O. Frostman

\title{
Über eine besondere Halbgruppe
}

\author{
Von Bengt Stolt
}

\section{§ 1. Einleitung}

In einer Arbeit von 1926 hat Suschkewitsch eine Halbgruppe behandelt, siehe [1]. ${ }^{1}$ Sie wird in folgender Weise definiert:

Es sei eine Menge (Komplex) von Elementen gegeben; ausserdem sei auch eine Verknüpfungsart (Operation) dieser Elemente definiert, mittelst der zwei beliebige (in bestimmter Ordnung genommene) Elemente miteinander komponiert werden können, so dass sich ein bestimmtes Element als Resultat (,Produkt") dieser Komposition herausstellt. Diese Elementenmenge insgesamt mit der Operation soll nun folgenden Grundpostulaten unterliegen:

I. Die Operation ist eindeutig und unbeschränkt anwendbar.

II. Es gilt für sie das assoziative Gesetz.

III. Die Menge enthält nur eine endliche Anzahl von Elementen.

$\mathrm{IV}_{l}$. Es gilt das linke Gesetz der eindeutigen (also nach III auch der unbeschränkten) Umkehrbarkeit, d.h.: Aus der Gleichung $B A=C A$ folgt $B=C$. ([1], S. 32-33.)

Das obige System wird „eine endliche Gruppe ohne das Gesetz der eindeutigen Umkehrbarkeit" genannt, und ihre Eigenschaften werden eingehend studiert.

In 1946 hat Prachar eine Halbgruppe studiert; siehe [2]. Er geht von der folgenden Definition einer Gruppe aus:

Eine Gruppe ist eine Menge $G$ von Elementen, welche folgende Forderungen erfüllt:

1. Zu jedem geordneten Elementepaar $a \subset G, b \subset G$ existiert ein eindeutig bestimmtes drittes Element $c \subset G$, welches das Produkt von $a$ und $b$ heisst und mit $a b$ bezeichnet wird.

2. Das in 1. definierte Produkt ist assoziativ: für $a \subset G, b \subset G, c \subset G$ gilt

$$
a(b c)=(a b) c .
$$

3. Es existiert mindestens ein Element $e$, so dass gilt:

für alle $a \subset G$.

$$
e a=a
$$

4. $\mathrm{Zu}$ jedem $a \subset G$ existiert mindestens ein Element $a^{-1}$, für das die Gleichung

besteht.

$$
a^{-1} a=e
$$

\footnotetext{
1 Die Zahlen in eckigen Klammern beziehen sich auf das Literaturverzeichnis am Schluss die-
} ser Arbeit. 


\section{B. stoLt, Über eine besondere Halbgruppe}

Daraus ergeben sich dann die Folgerungen:

5. Für jedes $a$ gilt auch die Gleichung:

$$
a a^{-1}=\text { e. ([2] S. 97.) }
$$

Prachar studiert nun alle Systeme $S$ mit endlich vielen Elementen, welche 1., 2., 3., 5 erfüllen. Seine Arbeit scheint ohne Kenntnis der Arbeit Suschkewitschs geschrieben zu sein. Es leuchtet nämlich ein, dass die Halbgruppen von Suschkewitsch und Prachar identisch sind.

Es ist das Ziel vorliegender Arbeit, die Identität dieser Halbgruppen zu zeigen. Diese Untersuchung ist um so mehr von Interesse, als die Halbgruppe in der Gruppenaxiomatik sehr oft als Gegenbeispiel benutzt wird.

Zunächst werden wir eine Halbgruppe $S$ auf eine dritte Art definieren. Dann werden wir ihre Eigenschaften bestimmen und die einfachsten Beispiele solcher Halbgruppen aufstellen. Schliesslich werden wir eine axiomatische Untersuchung vornehmen, wobei sämtliche irreduzible Axiomensysteme aufgestellt werden, die $S$ charakterisieren. Es wird gezeigt, dass die Definitionen von Suschkewitsch und Prachar auf solche Axiomensysteme zurückgeführt werden können.

\section{$\S 2$. Definition und Eigenschaften der Halbgruppe $S$}

Ehe wir die Halbgruppe $S$ definieren, werden wir zunächst an die folgende Definition einer Gruppe erinnern.

Eine abstrakte Gruppe kann als eine nichtleere Menge definiert werden, in welcher die folgenden vier Axiome erfüllt sind.

1. Es ist eine Zusammensetzungsvorschrift gegeben, so dass jedem geordneten Paar $a, b$ von Elementen der Menge eindeutig ein drittes Element der Menge zugeordnet ist, in Formel $a b=c$.

2. Für drei beliebige Elemente $a, b$ und $c$ besteht das assoziative Gesetz: $(a b) c=$ $a(b c)$.

3. Zu zwei beliebigen Elementen $b, c$ der Menge gibt es genau ein Element $a$, das $a b=c$ erfüllt.

4. Zu zwei beliebigen Elementen $a, c$ der Menge gibt es genau ein Element $b$, das $a b=c$ erfüllt.

Wir definieren nun die Halbgruppe $S$ als eine Menge, in welcher nur die Axiome 1.-3. aber nicht das Axiom 4. erfüllt sind. $S$ kann endlich oder unendlich sein.

Wir werden nun die folgenden Sätze beweisen.

Satz 1. $Z u$ jedem Elemente a aus $S$ gibt es genau ein Element $e_{a}$, das $e_{a} a=a$ erfïllt. Ferner ist $e_{a}$ ein rechtsseitiges Einselement.

Beweis: Für jedes a aus $S$ folgt unmittelbar die Existenz eines $e_{a}$ mit $e_{a} a=a$ aus 3 . Aus

folgt $e_{a} e_{a}=e_{a}$ aus 3 .

$$
\left(e_{a} e_{a}\right) a=e_{a}\left(e_{a} a\right)=e_{a} a=a
$$

Wenn $b$ ein beliebiges Element ist, gilt wegen $3 . b_{1} e_{a}=b$. Aus

$$
b e_{a}=\left(b_{1} e_{a}\right) e_{a}=b_{1}\left(e_{a} e_{a}\right)=b_{1} e_{a}=b
$$

folgt, dass $e_{a}$ ein rechtsseitiges Einselement ist. Hiermit ist der Satz bewiesen. 
Aus diesem Satz folgt unmittelbar

Satz 2. In S gibt es mindestens zwei rechtsseitige Einselemente.

Beweis: Aus dem vorigen Satz geht hervor, dass man zu jedem $a$ aus $S$ ein $e_{a}$ bestimmen kann, das $e_{a} a=a$ erfüllt und ein rechtsseitiges Einselement ist. Wenn es in $S$ nur ein rechtsseitiges Element gäbe, wären alle $e_{a}$ untereinander gleich. Folglich bestände ein linksseitiges Einselement, was gegen die Voraussetzung ist.

Wir werden nun die Elemente von $S$ in Klassen einteilen.

$Z$ wei Elemente $b$ und $c$ gehören zu derselben Klasse, wenn die Gleichung $a b=a c$ für jedes a erfüllt ist.

Es ist klar, dass alle rechtsseitigen Einselemente eine Klasse bilden. Denn für zwei solche Elemente $e_{i}$ und $e_{j}$ besteht ja immer $a e_{i}=a e_{j}=a$, wo $a$ beliebig ist. Die Klasse aller rechtsseitigen Einselemente werden wir die Einheitsklasse nennen und mit $E$ bezeichnen. Es leuchtet unmittelbar ein, dass $E$ nur rechtsseitige Einselemente enthält.

Zunächst werden wir drei Lemmata beweisen.

LEMMa 1. Wenn $e_{i}$ und $e_{j}$ zwei rechtsseitige Einselemente sind, $e_{i} \neq e_{j}$, so gilt $e_{i} a \neq e_{j}$ fiir jedes beliebige a.

Beweis: Sonst bestände

$$
e_{j}=e_{i} a=\left(e_{i} e_{i}\right) a=e_{i}\left(e_{i} a\right)=e_{i} e_{j}=e_{i},
$$

was gegen die Voraussetzung ist.

LemMa 2. Es sei $e_{i}$ ein rechtsseitiges Einselement. Wenn $e_{i} g=e_{i}$ für ein gewisses Element $g$ gilt, so ist auch $g$ ein rechtsseitiges Einselement.

Beweis: Für jedes beliebige $a$ gilt

$$
a g=\left(a e_{i}\right) g=a\left(e_{i} g\right)=a e_{i} .
$$

Nach der obige Definition gehören folglich $g$ und $e_{i}$ zu derselben Klasse, wodurch das Lemma bewiesen ist.

Lemma 3. Es seien $b$ und $c$ gewisse Elemente aus $S$ und $e_{i}$ ein rechtsseitiges Einselement, das $e_{i} b=c$ erfüllt. Dann gehören $b$ und $c$ zu derselben Klasse.

Beweis: Wenn a beliebig ist, so gilt

$$
a b=\left(a e_{i}\right) b=a\left(e_{i} b\right)=a c,
$$

wodurch das Lemma bewiesen ist.

Es ist nun möglich, den folgenden Satz zu beweisen.

Satz 3. Wenn die Halbgruppe $S$ genau $m$ rechtsseitige Einselemente enthält, zerfällt sie in Klassen mit je genau $m$ Elementen. Jedes Element von $S$ gehört genau einer Klasse. 


\section{B. STOLT, Über eine besondere Halbgruppe}

Beweis: Wir nehmen an, dass $e_{1}, e_{2}, \ldots, e_{m}$ die Elemente der Einheitsklasse sind. Es sei $a_{1}$ ein gewisses Element, das zur Einheitsklasse nicht gehört. Wegen Axiom 3. gibt es ein Element $e_{1}$ der Einheitsklasse, das $e_{1} a_{1}=a_{1}$ erfüllt. Wegen Axiom 1. ist es möglich, der Reihe nach die folgenden Produkte zu bilden. Bei geeigneter Numerierung der Produkte erhalten wir

$$
\begin{aligned}
& e_{1} a_{1}=a_{1} \\
& e_{2} a_{1}=a_{2} \\
& \cdots \\
& e_{m} a_{1}=a_{m}
\end{aligned}
$$

Es ist klar, dass alle $a_{j}$ verschieden sind. Denn wäre $e_{i} a_{1}=e_{j} a_{1}=a_{j}$, so wäre $e_{i}=e_{j}$, was gegen die Voraussetzung ist. Weil $a_{1}$ kein rechtsseitiges Einselement ist, gehört kein $a_{j}$ zur Einheitsklasse. Den wäre $e_{i} a_{1}=e_{j}$, so folgte $e_{j}=e_{i}$ aus Lemma 1 , und wegen Lemma 2 wäre dann $a_{1}$ ein rechtsseitiges Einselement, was gegen die Voraussetzung ist. Wegen Lemma 3 ist klar, dass alle $a_{j}$ zu derselben Klasse $A$ hören.

Für die in obiger Weise numerierten Elemente gilt allgemein

Denn man erhält leicht

$$
e_{i} a_{j}=a_{i}
$$

$$
e_{i} a_{j}=e_{i}\left(e_{j} a_{1}\right)=\left(e_{i} e_{j}\right) a_{1}=e_{i} a_{1}=a_{i} .
$$

Es bleibt übrig zu zeigen, dass $A$ nur die $m$ Elemente $a_{1}, a_{2}, \ldots, a_{m}$ enthält. Denn wäre $b$ ein Element, dass zu $A$ gehörte, so bestände $a b=a a_{j}$ für jedes $a$ aus $S$. Für $a=e_{i}$ folgte dann $e_{i} b=e_{i} a_{j}=a_{i}$. Wenn $e_{i}$ sämtliche Elemente von $E$ durchläuft, durchläuft folglich das Produkt $e_{i} b$ sämtliche Elemente $a_{1}, a_{2}, \ldots, a_{m}$. Aber ein Element aus $E$ muss die Gleichung $x b=b$ erfüllen. Dieses ist nur möglich, wenn $b$ mit einem gewissen $a_{j}$ identisch ist.

Sei ferner $b_{1}$ ein Element aus $S$, das nicht zu $E$ oder $A$ gehört. Dann erhalten wir bei passender Numerierung:

$$
\begin{gathered}
e_{1} b_{1}=b_{1} \\
e_{2} b_{1}=b_{2} \\
\cdots \\
e_{m} b_{1}=b_{m}
\end{gathered}
$$

Sämtliche Elemente $b_{1}, b_{2}, \ldots, b_{m}$ bilden offenbar eine Klasse, die wir $B$ nennen. Es ist klar, dass wir in dieser Weise sämtliche Elemente der Halbgruppe in Klassen einteilen können, die untereinander kein Element gemeinsam haben. Folglich ist der Satz bewiesen.

Aus diesem Satz folgt unmittelbar.

Satz 4. Wenn die Anzahl der Elemente $n$ von $S$ endlich ist, so ist die Anzahl $m$ der rechtsseitigen Einselemente ein Teiler von $n$.

Aus der Numerierung der Elemente, die wir in Satz 3 vorgenommen haben, folgt nun Lemma 4.

Lemma 4. Die Gleichung

$$
x b_{j}=c_{k}
$$

kann nur von einem Element mit dem Index $k$ erfüllt werden. 
ARKIV FÖR MATEMATIK. Bd 3 nr 23

Beweis: Es ist klar, dass es in $S$ genau ein Element $a_{i}$ gibt, das $a_{i} b_{j}=c_{k}$ erfüllt. Aus $e_{i} a_{i}=a_{i}$ ergibt sich dann

woraus $i=k$ folgt.

$$
c_{k}=a_{i} b_{j}=\left(e_{i} a_{i}\right) b_{j}=e_{i}\left(a_{i} b_{j}\right)=e_{i} c_{k}=c_{i}
$$

Wir beweisen auch

Lemma 5. Die Produkte von Elementen der Klasse $A$ und Elementen der Klasse $B$ gehören zu derselben Klasse $C$.

Beweis: Es seien $a_{i}$ und $a_{h}$ zwei Elemente aus $A$ und $b_{j}$ und $b_{k}$ zwei Elemente aus $B$. Wegen Lemma 4 gilt $a_{i} b_{j}=c_{i}, a_{h} b_{k}=d_{h}$. Hieraus ergibt sich

$$
c_{i}=a_{i} b_{j}=\left(e_{i} a_{h}\right)\left(e_{j} b_{k}\right)=e_{i}\left(a_{h} e_{j}\right) b_{k}=e_{i}\left(a_{h} b_{k}\right)=e_{i} d_{h}=d_{i} .
$$

Dadurch ist gezeigt, dass $c_{i}$ und $d_{h}$ zu derselben Klasse gehören.

Das Produkt der Klassen $A$ und $B$ definieren wir als die Gesamtheit der Produkte $a_{i} b_{j}=c_{i}$ aller Elemente $a_{i}$ der Klasse $A$ und aller Elemente $b_{j}$ der Klasse $B$. Aus den bisherigen Überlegungen leuchtet ein, dass alle Produkte $c_{i}$ eine Klasse $C$ bilden. Es gilt folglich $A B=C$.

Das Produkt $a_{i} B$ von einem Element $a_{i}$ und einer Klasse $B$ wird in analoger Weise als die Gesamtheit der Produkte $a_{i} b_{j}=c_{i}$ definiert, wo $b_{j}$ die Elemente der Klasse $B$ durchläuft. Aus den vorigen Lemmata leuchtet ein, dass

gilt.

$$
a_{i} B=c_{i}
$$

Wir beweisen nun

Lamma 6. Die Gesamtheit von Lösungen $x$ der Gleichungen

$$
x b_{i}=c_{h},
$$

wo $b_{i}$ die Elemente der Klasse $B$ und $c_{h}$ die Elemente der Klasse $C$ durchlaufen, gehören $z u$ derselben Klasse $X=A$.

Beweis: Wegen Lemma 4 ist klar, dass die Gleichung

$$
x b_{i}=c_{h}
$$

von einem Element $x=a_{h}$ erfüllt ist. Wenn $b_{i}$ und $c_{h}$ die Klassen $B$ bzw. $C$ durchlaufen, werden wir zeigen, dass alle Elemente zu derselben Klasse gehören. Denn bestände $a_{h} b_{i}=c_{h}$ und $d_{k} b_{j}=c_{k}$, so wäre

$$
a_{k} b_{j}=\left(e_{k} a_{h}\right)\left(e_{j} b_{i}\right)=e_{k}\left(a_{h} e_{j}\right) b_{i}=e_{k}\left(a_{h} b_{i}\right)=e_{k} c_{h}=c_{k},
$$

woraus $d_{k}=a_{k}$ folgt. Die Gleichung $X B=C$ ist also von einer gewissen Klasse $X=A$ erfüllt.

Mit Hilfe der Komposition von Klassen ist es möglich, den folgenden wichtigen Satz zu bewiesen.

Satz 5. Die Klassen von $S$ bilden eine Gruppe G. 


\section{B. sтoLt, Über eine besondere Halbgruppe}

Beweis: Für die Komposition von Klassen ist klar, dass die Axiome 1.-3. bestehen.

Wenn $e_{i}$ die Elemente der Einheitsklasse $E$ und $a_{j}$ die Elemente einer beliebigen klasse $A$ durchlaufen, durchläuft das Produkt $e_{i} a_{j}=a_{i}$ sämtliche Elemente von $A$. Also gilt $E A=A$, woraus folgt, dass $E$ ein linksseitiges Einselement von $G$ ist. Die Klassen von $S$ bilden folglich eine Gruppe $G$, womit der Satz bewiesen ist.

Die folgenden Sätze gehen aus den bisher bewiesenen Sätzen hervor.

Satz 6. Die Halbgruppe $S$ ist völlig bestimmt, wenn man die Gruppe G der Klassen und die Anzahl $m$ der rechtsseitigen Einselemente von $S$ kennt.

Satz 7. Die Untermenge aller Elemente aus $S$ mit demselben Index $i$ ist eine Gruppe $G_{i}$, die mit der Gruppe $G$ der Klassen isomorph ist. Ferner gilt $e_{i} G=G_{i}$, wo $e_{i} d a s$ rechtsseitige Einselement ist.

Satz 8. Wenn $G$ die Gruppe der Klassen von $S$ und $e_{1}, e_{2}, \ldots, e_{m}$ die rechtsseitigen Einselemente von $S$ sind, ist es möglich, die Halbgruppe $S$ in folgender Weise als eine Summe isomorpher Gruppen zu schreiben.

$$
S=e_{1} G+e_{2} G+\cdots+e_{m} G .
$$

Wenn die Halbgruppe $S$ endlich ist, ist eine analoge Formel ohne Benutzung des Klassenbegriffes von Suschkewitsch und Prachar gefunden. In einem Referat in den Mathematical Reviews 9 (1948) S. 491 vermutet Jennings, dass der Fall einer unendlichen Halbgruppe schwieriger ist. Es leuchtet aber ein, dass die Sätze vorliegender Arbeit sowohl für endliche Halbgruppen $S$ als auch für unendliche Halbgruppen $S$ mit einer endlichen Anzahl rechtsseitiger Einselemente hergeleitet worden sind.

\section{§ 3. Beispiele von Halbgruppen $S$}

In diesem Abschnitt werden wir die einfachsten Beispiele der Halbgruppe $S$ konstruieren. Die Elemente von $S$ werden mit den Ziffern 1, $2, \ldots$ bezeichnet. $n$ sei die Anzahl der Elemente, $m$ die Anzahl der rechtsseitigen Einselemente.

Das einfachste Beispiel ist $m=n=2$, das in gruppenaxiomatischer Literatur als Gegenbeispiel sehr oft benutzt wird.

$$
\begin{array}{ll}
1 & 1 \\
2 & 2
\end{array}
$$
ist.

Wenn $n=p$ eine Primzahl ist, so leuchtet ein, dass nur der Fall $m=n=p$ möglich

Das einfachste Beispiel mit $m \neq n$ ist $n=4, m=2$, das in folgender Weise geschrieben werden kann.

$\begin{array}{llll}1 & 1 & 4 & 4 \\ 2 & 2 & 3 & 3 \\ 3 & 3 & 2 & 2 \\ 4 & 4 & 1 & 1\end{array}$

Für $n=6$ erhalten wir drei Halbgruppen $S$, nämlich $m=6$ und $m=3$, die mit den vorigen Beispielen analog sind, und $m=2$, wobei die Klassen eine zyklische Gruppe von der Ordnung 3 bilden. 
ARKIV För MATEMATIK. Bd 3 nr 23

$\begin{array}{llllll}\mathbf{1} & \mathbf{1} & \mathbf{3} & \mathbf{3} & \mathbf{5} & \mathbf{5} \\ \mathbf{2} & \mathbf{2} & \mathbf{4} & \mathbf{4} & \mathbf{6} & \mathbf{6} \\ \mathbf{3} & \mathbf{3} & 5 & \mathbf{5} & \mathbf{1} & \mathbf{1} \\ \mathbf{4} & \mathbf{4} & \mathbf{6} & \mathbf{6} & \mathbf{2} & \mathbf{2} \\ \mathbf{5} & \mathbf{5} & \mathbf{1} & \mathbf{1} & \mathbf{3} & \mathbf{3} \\ \mathbf{6} & 6 & 2 & \mathbf{2} & \mathbf{4} & \mathbf{4}\end{array}$

Für $n=8$ sind vier Halbgruppen möglich. Wenn $m=8$ oder $m=4$ ist, erhalten wir Halbgruppen von schon behandelten Typen. Für $m=2$ gibt es die folgenden zwei Halbgruppen, die der zyklischen Gruppe der Ordnung 4 und der Vierergruppe entsprechen.

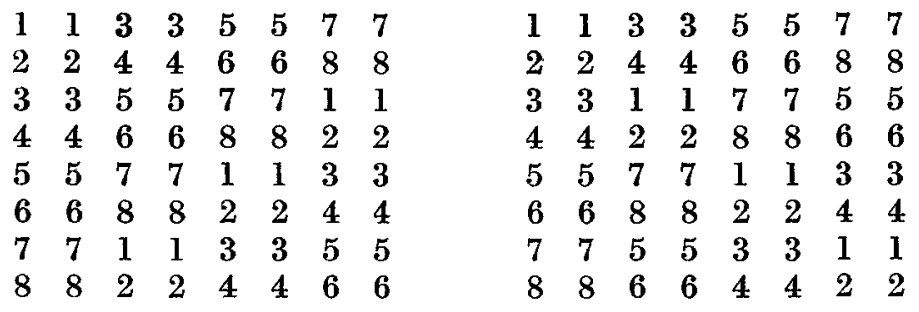

Schliesslich teilen wir eine Tafel der Anzahl aller verschiedenen Halbgruppen $S$ mit gegebener Anzahl von Elementen mit, $n \leqq 25$.

\begin{tabular}{|c|c|c|c|}
\hline $\begin{array}{c}\text { Anzahl der } \\
\text { Elemente }\end{array}$ & $\begin{array}{c}\text { Anzahl der } \\
\text { Halbgruppen }\end{array}$ & $\begin{array}{c}\text { Anzahl der } \\
\text { Elemente }\end{array}$ & $\begin{array}{c}\text { Anzahl der } \\
\text { Halbgruppen }\end{array}$ \\
\hline & 1 & 14 & 3 \\
2 & 1 & 15 & 3 \\
3 & 2 & 16 & 9 \\
4 & 1 & 17 & 1 \\
5 & 3 & 18 & 7 \\
6 & 1 & 19 & 1 \\
7 & 4 & 20 & 7 \\
8 & 2 & 21 & 3 \\
9 & 3 & 22 & 3 \\
10 & 1 & 23 & 1 \\
11 & 7 & 24 & 17 \\
12 & 1 & 25 & 2 \\
13 & & & \\
\hline
\end{tabular}

\section{§ 4. Axiomatische Untersuchung}

Wir wollen schliesslich eine axiomatische Untersuchung vornehmen. Zunächst stellen wir einige Axiome auf, die von Baer-Levi [3], Lorenzen [4] und Stolt [5] eingeführt worden sind. Dann werden wir sämtliche vollständigen irreduziblen Systeme aufstellen, die aus diesen Axiomen gebildet werden können. Zum Schluss wird gezeigt, dass nur die gegebenen Systeme vollständig und irreduzibel sind.

Zugrunde dieser Untersuchung liegt eine nichtleere Menge, in welcher eine dreistellige Relation $a b \supset c$ definiert ist, wo $a, b$ und $c$ Elemente der Menge sind.

Die betrachteten Axiome sind wie folgt. 
B. STоLт, Über eine besondere Halbgruppe

Assoziativaxiom.

A: Wenn zu drei Elementen $a, b$ und $c$ die Produkte $(a b) c$ und $a(b c)$ existieren, bestimmen sie denselben Wertevorrat.

Allgemeine Existenz- und Unitätsaxiome.

E: Zu je zwei Elementen $a$ und $b$ gibt es mindestens ein $c$, das $a b \supset c$ erfüllt.

IE: Zu je zwei Elementen $b$ und $c$ gibt es mindestens ein $a$, das $a b \supset c$ erfüllt.

$\mathrm{U}: \mathrm{Zu}$ je zwei Elementen $a$ und $b$ gibt es höchstens ein $c$, das $a b \supset c$ erfüllt.

IU: Zu je zwei Elementen $b$ und $c$ gibt es höchstens ein $a$, das $a b \supset c$ erfüllt.

Eins-Existenzaxiome.

rI: Es gibt mindestens ein Element $e$, das $a e \supset a$ erfüllt, wo a beliebig ist.

I: Es gibt mindestens ein Element $e$, das $e e \supset e$ erfüllt.

li: Es gibt mindestens ein Element $c$, zu welchem es mindestens ein $e$ gibt, das $e c \supset c$ erfüllt.

ri: Es gibt mindestens ein Element $c$, zu welchem es mindestens ein $e$ gibt, das $c e \supset c$ erfüllt.

i: Es gibt mindestens ein Element $d$.

Mit $J$ wird eines der Axiome $r I, I, l i, r i$ oder $i$ bezeichnet.

\section{Allgemeine Inversaxiome.}

IE.J: Es gibt mindestens ein Element $e$ mit der Eigenschaft $J$. Zu jedem beliebigen $a$ gibt es mindestens ein $a^{\prime}$, das $a^{\prime} a \supset e$ erfüllt.

IU.J: Es gibt mindestens ein Element $e$ mit der Eigenschaft $J$. Zu jedem beliebigen $a$ gibt es höchstens ein $a^{\prime}$, das $a^{\prime} a \supset e$ erfüllt.

Spezielle Inversaxiome.

l€.J: Es gibt mindestens ein Element $e$ mit der Eigenschaft $J$. Zu mindestens ein Element $c$ gibt es mindestens ein $c^{\prime}$, das $c^{\prime} c \supset e$ erfültt.

lu.J: Es gibt mindestens ein Element $e$ mit der Eigenschaft $J$. Zu mindestens ein Element $c$ gibt es höchstens ein $c^{\prime}$, das $c^{\prime} c \supset e$ erfüllt.

Endlichkeitsaxiom.

N: In der Menge gibt es nur eine endliche Anzahl $n$ von Elementen.

Wenn in einem System mehrere Inversaxiome bestehen, wollen wir voraussetzen, dass sämtliche Inversaxiome für ein gewisses Einselement bestehen.

Wenn die Komposition der Menge eindeutig ist, ist $l U$ mit dem Axiom $I_{l}$ von Suschkewitsch identisch. Sonst ist $l U$ stärker als $\mathrm{IV}_{l}$.

Wir wollen nun die Vollständigkeit der folgenden Systeme zeigen.

1) $A, l E, U, r i$

2) $A, E, U, l E . r I$

3) $A, E, l U, l E . i$

4) $A, E, l E, U, l v . i$

5) $A, N, l E, U$

6) $A, N, E, l U$ 
ARKIV FÖR MATRMATIK. Bd 3 nr 23

Von diesen Systemen ist das System von Prachar mit 2. identisch, während das System von Suschkewitsch umfassender als 6. ist.

In den Vollständigkeitsbeweisen werden die Symbole $\ni$ (es existiert) und $\rightarrow$ (es folgt) verwendet.

Vollständigkeitsbeweis von 1.

$\ni c$ und $e$, die $c e=c$ erfüllen. $\ni a_{1} e=a$ und $g c=a_{1}$ wegen $l E, a$ beliebig. $\rightarrow$

$\rightarrow a e=a \rightarrow e$ mit $r I$.

$$
a=a_{1} e=(g c) e=g(c e)=g c=a_{1} \text {. }
$$

$\exists b^{\prime} b=e, b_{1} b^{\prime}=e, e_{1} b=b$ und $b_{2} b^{\prime}=e_{1}$ wegen $l E, b$ beliebig. $\rightarrow$

$$
b_{2}=b_{2} e=b_{2}\left(b^{\prime} b\right)=\left(b_{2} b^{\prime}\right) b=e_{1} b=b .
$$

$\rightarrow b b^{\prime}=e_{1}$. Zunächst werden wir beweisen,dass $e_{1}$ die Eigenschaft $r I$ hat und dass die Gültigkeit von $E$ hieraus folgt.

$$
\begin{aligned}
& \begin{array}{l}
\exists e^{\prime} e_{1}=e, e_{2} e^{\prime}=e, e_{3} e_{1}=e_{2} \text { und } e_{4} e^{\prime}=e_{3} \text { wegen } l E . \rightarrow \\
\qquad e_{4}=e_{4} e=e_{4}\left(e^{\prime} e_{1}\right)=\left(e_{4} e^{\prime}\right) e_{1}=e_{3} e_{1}=e_{2} . \\
\rightarrow e_{2} e^{\prime}=e_{3} \rightarrow e_{3}=e \text { wegen } U . \rightarrow e e_{1}=e_{2} . \\
\exists e_{5} b=b_{1} \text { und } b_{3} b^{\prime}=e_{5} \text { wegen } l E . \rightarrow \\
\qquad b_{3}=b_{3} e=b_{3}\left(b^{\prime} b\right)=\left(b_{3} b^{\prime}\right) b=e_{5} b=b_{1}
\end{array} \\
& \rightarrow b_{1} b^{\prime}=e_{5} \rightarrow e_{5}=e \rightarrow e b=b_{1} .
\end{aligned}
$$

Ferner bilden wir

$$
e_{2}=e e_{1}=e\left(b b^{\prime}\right)=(e b) b^{\prime}=b_{1} b^{\prime}=e .
$$

$\rightarrow e e_{1}=e \rightarrow a_{2} e_{1}=a$ wegen $l E . \rightarrow$

$$
a_{2}=a_{2} e=a_{2}\left(e e_{1}\right)=\left(a_{2} e\right) e_{1}=a_{2} e_{1}=a .
$$

$\rightarrow a e_{1}=a$, d.h. $e_{1}$ hat die Eigenschaft $r I$.

$\ni c_{1} b^{\prime}=a$ und $a_{3} b=c_{1}$ wegen $l E$, wo $a$ und $b$ beliebig sind. $\rightarrow$

$$
a_{3}=a_{3} e_{1}=a_{3}\left(b b^{\prime}\right)=\left(a_{3} b\right) b^{\prime}=c_{1} b^{\prime}=a
$$

$\rightarrow a b=c_{1}$, d.h. $E$ gilt.

Es bleibt übrig zu zeigen, dass $l U$ gilt. Sonst $\ni a_{1} b=c$ und $a_{2} b=c$ für mindestens ein Elementenpaar $b$ und $c . \rightarrow b b^{\prime}=e_{1}, c b^{\prime}=a_{1}$ und $c b^{\prime}=a_{2}$, was soeben bewiesen worden ist. $\rightarrow a_{2}=a_{1}$ wegen $U$, d.h. $l U$ gilt. Hiermit ist die Vollständigkeit bewiesen.

Vollständigkeitsbeweis von 2.

Wir werden dieses System auf 1. dadurch zurückführen, dass wir die Gültigkeit von $l E$ zeigen.

$\ni e$ mit $l E . r I . \rightarrow b^{\prime} b=e$ und $c b^{\prime}=a$, wo $b$ und $c$ beliebig sind. $\rightarrow$

$$
a b=\left(c b^{\prime}\right) b=c\left(b^{\prime} b\right)=c e=c .
$$




\section{B. StoLt, Über eine besondere Halbgruppe}

Hiermit ist 2. auf 1. zurückgeführt.

Vollständigkeitsbeweis von 3.

$\ni d$ mit $l E . i \rightarrow e d \supset d, f^{\prime} a \supset d, a$ beliebig. $\rightarrow$

$\rightarrow e e \supset e$ wegen $A, E$ und $l U$.

$$
(e e) d=e(e d) \supset e d \supset d
$$

$\ni a e \supset a_{1}$ wegen $E . \rightarrow$

$$
(a e) e=a(e e) \supset a e \supset a_{1}
$$

$\rightarrow a e \supset a_{2}, a_{2} e \supset a_{1}$ wegen $A$ und $E . \rightarrow a_{2}=a_{1}$ wegen $l U \rightarrow a e \supset a$. Folglich hat $e$ die Eigenschaft $r I$.

$\ni d d \supset d^{2}, d^{\prime} d^{2} \supset d . \rightarrow$

$$
\left(d^{\prime} d\right) d=d^{\prime}(d d) \supset d^{\prime} d^{2} \supset d .
$$

$\rightarrow d^{\prime} d \supset e$ wegen $A, E$ und $l U$. Wir bilden ferner

$$
\left(d^{\prime} f^{\prime}\right) a=d^{\prime}\left(f^{\prime} a\right) \supset d^{\prime} d \supset e .
$$

$\rightarrow d^{\prime} f^{\prime} \supset a^{\prime}, a^{\prime} a \supset e$ wegen $A, E$ und $l U . e$ hat also die Eigenschaft $l E . r I$.

Zunächst werden wir zeigen, dass $U$ gilt. Sonst $\ni a b \supset(c, g)$ für gewisse $a, b$. $\rightarrow b^{\prime} b \supset e, c^{\prime} c \supset e, g^{\prime} g \supset e$ wegen $\operatorname{lE} . r I . \rightarrow$

$$
\begin{aligned}
& \left(c^{\prime} a\right) b=c^{\prime}(a b) \supset c^{\prime} c \supset e \\
& \left(g^{\prime} a\right) b=g^{\prime}(a b) \supset g^{\prime} g \supset e .
\end{aligned}
$$

$\rightarrow c^{\prime} a \supset b^{\prime}, g^{\prime} a \supset b^{\prime}$ wegen $A, E$ und $l U . \rightarrow c^{\prime}=g^{\prime}$.

Wir bilden ferner

$$
\begin{aligned}
& \left(c c^{\prime}\right) c=c\left(c^{\prime} c\right) \supset c e \supset c, \\
& \left(g c^{\prime}\right) g=g\left(c^{\prime} g\right) \supset g e \supset g .
\end{aligned}
$$

$\rightarrow c c^{\prime} \supset e_{c}, e_{c} \supset c$ und $g c^{\prime} \supset e_{g}, e_{g} g \supset g$ wegen $A$ und $E . \rightarrow$

$$
\begin{aligned}
& \left(e_{c} a\right) b=e_{c}(a b) \supset e_{c} c \supset c, \\
& \left(e_{g} a\right) b=e_{g}(a b) \supset e_{g} g \supset g .
\end{aligned}
$$

$\rightarrow e_{c} a \supset a, e_{g} a \supset a$ wegen $A, E$ und $l U . \rightarrow e_{c}=e_{g}$ wegen $l U . \rightarrow c c^{\prime} \supset e_{c}, g c^{\prime} \supset e_{c} \rightarrow$ $c=g$ wegen $l U$. Folglich besteht $U$, womit 3. auf 2 . zurückgeführt ist.

Vollständigkeitsbeweis von 4.

$\ni c, d$ mit $h^{\prime} c=d$ wegen $l E$ und $l v . i . \rightarrow e c=c$ wegen $l E$. Wir bilden

$$
\left(h^{\prime} e\right) c=h^{\prime}(e c)=h^{\prime} c=d
$$

$\rightarrow h^{\prime} e=h^{\prime}$ wegen $A, E$ und $l v . i$. Dann besteht $r i$, womit 4 . auf. 1. zurückgeführt ist.

Vollständigkeitsbeweise von 5. und 6 .

Diese Beweise sind mit den Beweisen von Baer-Levi [3], S. 11, und Stolt [6], S. 114, völlig analog. Wir werden uns auf den Fall beschränken, wo $E$ und $l U$ bestehen und betrachten die Produkttafel der zugrundeliegenden endlichen Menge. Wenn $E$ besteht, gibt es in jeder Parzelle der Produkttafel mindestens ein Element. 
ARKIV För MATEMATIK. Bd 3 nr 23

Eine beliebige Kolonne enthält folglich mindestens $n$ Elemente. Aber wegen $l U$ kommt jedes Element in jeder Kolonne höchstens einmal vor. Dann müssen sämtliche $n$ Elemente in jeder Kolonne genau einmal vorkommen, d.h. $l E$ besteht. Weil jede Parzelle genau ein Element enthalten muss, besteht auch $U$.

Der Beweis für den Fall, wo $l E$ und $U$ bestehen, ist derselbe, wenn $E$ durch $U$ und $l U$ durch $l E$ ersetzt und die Worte ,,mindestens" und ,,höchstens" miteinander vertauscht werden. Hiermit ist die Vollständigkeit von 5. und 6. gezeigt.

Es bleibt übrig zu zeigen, dass es nur die obigen vollständigen Systeme gibt. Mit Hilfe folgender unvollständiger Systeme ist dies leicht zu bestätigen. Es leuchtet ferner ein, dass die vollständigen Systeme irreduzibel sind, d.h. dass kein Axiom durch ein schwächeres Axiom ersetzt werden kann.

Die unvollständigen Systeme sind wie folgt.
1) $N, E, l E, U, l U, r I$
2) $A, N, l E, l U, r I$
3) $A, N, U, l U, l E . r I$
4) $A, N, E, l E, l U . r I$
5) $A, N, E, U, l E . I, l U . I$
6) $A, E, U, l U, l \varepsilon . r I$
7) $A, E, l E, U, l i$
8) $A, l E, U, l U, l i$

Die Unvollständigkeit der Systeme 1.-6. geht aus den folgenden Beispielen hervor.

1.

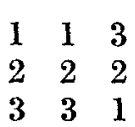

3.

$\begin{array}{lll}1 & 1 & 1 \\ 2 & 2 & 2 \\ 3 & 3 & -\end{array}$

2.

$\begin{array}{llc}1 & 1 & - \\ 2 & 2 & - \\ 3 & 3 & (1, \overline{2}, 3)\end{array}$

4.

$\begin{array}{ccc}1 & 1 & 1 \\ 2 & 2 & 2 \\ (2,3) & (2,3) & (2,3)\end{array}$

5.
$\begin{array}{lll}1 & 1 & 1\end{array}$
$\begin{array}{lll}2 & 2 & 2\end{array}$

6.

$\begin{array}{rrrrrrrrrrr}1 & 1 & 3 & 3 & 5 & 5 & 7 & 7 & 9 & 9 & \ldots \\ 2 & 2 & 4 & 4 & 6 & 6 & 8 & 8 & 10 & 10 & \\ 3 & 3 & 7 & 7 & 11 & 11 & 15 & 15 & 19 & 19 & \\ 4 & 4 & 8 & 8 & 12 & 12 & 16 & 16 & 20 & 20 & \\ 5 & 5 & 11 & 11 & 17 & 17 & 23 & 23 & 29 & 29 & \\ 6 & 6 & 12 & 12 & 18 & 18 & 24 & 24 & 30 & 30 & \\ 7 & 7 & 15 & 15 & 23 & 23 & 31 & 31 & 39 & 39 & \\ 8 & 8 & 16 & 16 & 24 & 24 & 32 & 32 & 40 & 40 & \\ 9 & 9 & 19 & 19 & 29 & 29 & 39 & 39 & 49 & 49 & \\ 10 & 10 & 20 & 20 & 30 & 30 & 40 & 40 & 50 & 50 & \ldots\end{array}$


B. stoLt, Über eine besondere Halbgruppe

Die Unvollständigkeit von 7. geht aus [3] S. 7, [4] S. 320 oder [5] S. 51, von 8. aus [4] S. 320 oder [5] S. 32 hervor.

\section{I T E R A T U R}

[1]. A. SUschkewrisch, Über die endlichen Gruppen ohne das Gesetz der eindeutigen Umkehrbarkeit. Math. Ann. 99 (1928) 30-50.

[2]. K. Prachar, Zur Axiomatik der Gruppen. Akad. Wiss. Wien, S.-B. IIa, 155 (1947), 97-102.

[3]. R. BAER u. F. LEvi, Vollstêndige irreduzible Systeme von Gruppenaxiomen. S.-B. Heidelberger Akad. Wiss., Math.-Nat. Kl., 2. Abhandlung 1932, 3-12.

[4]. P. LorenzeN, Ein Beitrag zur Gruppenaxiomatik. Math. Z. 49 (1943-44), 313-327.

[5]. B. StoLt, Über Axiomensysteme, die eine abstrakte Gruppe bestimmen. Uppsala 1953.

[6]. — Über irreduzible Axiomensysteme, die eine endliche abstrakte Gruppe bestimmen. Ark. Mat. 3 (1954), 113-115. 